\title{
A clinical study on expectant management versus induction of labour in term premature rupture of membranes (PROM)
}

\begin{abstract}
Premature rupture of membranes (PROM), also called Prelabour Rupture Of Membranes, is classically defined as rupture of membranes before labour and accounts for $0.8-0.9 \%$ of all pregnancies at term. This study was conducted over a period of 14 months (from March 2015 to May 2016) in a tertiary care medical college in North India. A total of 100 term PROM patients were recruited in our study - divided into two groups randomly--50 (Group A) patients were managed conservatively and 50 (Group- B) patients underwent induction of labour. Both these patient groups were studied to compare the feto-maternal outcome. Group-A (conservative management group) patients were observed to await the spontaneous onset of labour pains for at least 24 hours. Patients in group B were induced with either - PGE1 tab (misoprostol) $25 \mu \mathrm{gm} 4$ hourly orally or iv oxytocin infusion. The PROM-delivery interval was $<12$ hours in $72 \%$ of induced groups (Group B) and $10 \%$ (5) in group- A (conservative or expectant group). LSCS rate was $10 \%$ in group-A (expectant group) \& $15 \%$ in group -B (induced group). Sepsis rate, maternal and fetal, hospital stay, NICU admission \& duration of NICU stay were notably higher in group - A (expectant management group). Therefore, from our study we concluded that immediate induction of labour in term PROM cases shortens the PROM- delivery interval, hospital stay, NICU stay and reduction in both maternal \& neonatal sepsis.
\end{abstract}

Keywords: Term PROM, Induction of labour, Feto-maternal outcome, Neonatal sepsis, NICU, Expectant management
Volume 5 Issue 3 - 2016

\section{Jayati Nath}

Department of Obstetrics \& Gynecology, Maharishi Markandeshwar Institute of Medical Sciences and Research, India

Correspondence: Jayati Nath, Associate Professor, Obstetrics \& Gynecology, Maharishi Markandeshwar Institute of Medical Sciences and Research, Ambala, Haryana-133207, India, Email jayati.nath@yahoo.com

Received: September II, 2016 | Published: October 04, 2016
Abbreviations: PROM, Premature Rupture Of Membranes; NICU, Neonatal Intensive Care Unit; PGE1, Prostaglandin E1; LSCS, Lower Segment Cesarean Section

\section{Introduction}

Premature or Prelabour Rupture Of Membranes is classically defined as the rupture of integrity of the fetal membranes before the onset of labour and resulting in leakage of amniotic fluid. ${ }^{1-4}$ Pre-labour rupture of membranes without spontaneous uterine contractions complicate approximately $10 \%$ of all pregnancies, out of which $80 \%$ occurs at term. ${ }^{5}$ The management of PROM at term remains a matter of great debate till date. While induction of labour has resulted in decreased incidence of maternal \& fetal sepsis, but it is also associated with a higher incidence of caesarean section rate due to fetal distress and uterine hyperstimulation. Kappa et $a .^{6}{ }^{6}$ in their study, reported a higher incidence of operative deliveries in term pregnancies with ruptured membranes managed with labour stimulations compared with those who were managed expectantly. ${ }^{6}$ Approximately $65-75 \%$ of term PROM patients are followed by spontaneous onset of labour within 24 hours. ${ }^{7}$ Prostaglandins- PGE2 \& PGE1 have been used for cervical ripening and myometrial stimulation in unfavorable cervices with low Bishop's pre- induction score. It is seen that in patients who had expectant management, with prolonged hospitalization without active intervention with uncertain fetal and neonatal prognosis, many a times leads to maternal psychological sequalae. ${ }^{8}$

\section{Materials \& methods}

Study type: This was a hospital based prospective observational study.
Study duration: Fourteen months

Study period: March 2015 to May 2016

Study subjects: women with term PROM as per inclusion \& exclusion criteria

\section{a. Inclusion criteria}

i. Gestational age $37-40$ weeks

ii. Singleton pregnancy

iii. Age 20-30 years

iv. Adequate pelvis

v. Vertex presentation

\section{b. Exclusion criteria}

I. Age $<20$ yrs , $>30$ yrs

II. Gestational age $<37$ weeks, $>40$ weeks

III. Multiple pregnancy

IV. Chorioamnionitis

V. Medical disorders

VI. Obstetric complications

VII. Grand multipara

VIII. Previous LSCS

IX. Meconium stained liquour 


\section{Cephalopelvic disproportion}

\section{Malpresentation}

\section{Intrauterine fetal death}

All the patients who fulfilled the inclusion and exclusion criteria were subjected to a detailed history taking followed by thorough clinical examination including general and systemic examination to rule out any exclusion criteria. A thorough obstetrical examination including per speculum examination and Bishop's scoring was conducted. An obstetric ultrasonography and CTG were performed. All the patients were given IV antibiotics of 1 gram ceftriaxone, 100 $\mathrm{ml}$ metronedazole and gentamycin $80 \mathrm{mg}$ after negative skin test till delivery. Informed written consent was taken and patients were randomly allocated to either Group A (expectant or conservative management group) or Group B (active management group with induction of labour).

Patients in group-B (active management group) were induced according to Bishop's pre-induction score. PGE1 tablet (misoprostol) $25 \mu \mathrm{m}$ orally was given if Bishop's score was less than 5 followed by repeat dose after 4 hours or iv oxytocin at the rate of 5 units in $500 \mathrm{ml}$ RL or N/S (for primigravida) or @ 1- 2 units for multigravida) and the drip was titrated in escalating doses till optimal response was observed as evidenced by onset of effective uterine contractions. All these patients had vitals charting, pulse, temperature, blood pressure along with progress of labour-fetal heart rate, uterine contractions and decent of head.
Patients in group-A (expectant management group) were kept for observation with sterile vulval pad for 24 hours to await spontaneous onset of labour pains. Vitals charting (pulse rate, temperature, blood pressure), uterine contractions fetal heart rate, colour of liquour were monitored carefully. Unnecessary vaginal examinations were avoided and $\mathrm{P} / \mathrm{V}$ exam done only if the uterine contractions were good, to gauge the progress of labour. In this group, most of the patients went into spontaneous labor in 24 hours. Induction of labour was done after 24 hours if there were no contraction. Emergency LSCS was done for indications like fetal distress, non progression of labour, cord prolapse, failed induction, and chorioamnionitis.

All labour and delivery events were noted example vaginal delivery, spontaneous or instrumental-forceps/ventouse, LSCS etc. The fetal outcomes were noted namely APGAR score, birth weight, neonatal sepsis, NICU admissions and care required. All the patients were followed up in puerperium to assess maternal pyrexia, sepsis and neonatal sepsis.

\section{Results}

The results and observations of the present study are as follows:

Most of the patients were primigravidae, had low education levels, hailed from rural background and from low socio-economic stratum, young aged and mostly unbooked.

Table I Distribution of patients according to parity

\begin{tabular}{lllll}
\hline & Group -A (Expectant & management) & Group- B(Induced) & (In) \\
& number & $\%$ & number & $\%$ \\
Primigravida & 27 & 54.0 & 22 & 44.0 \\
Multigravida & 23 & 46.0 & 28 & 56.0 \\
\hline Total & 50 & 100.0 & 50 & 100.0 \\
\hline
\end{tabular}

Table 2 Mode of delivery

\begin{tabular}{lllll}
\hline & \multicolumn{2}{l}{$\begin{array}{l}\text { Group - A ( Expectant) } \\
\text { number }\end{array}$} & $\%$ & \multicolumn{2}{l}{$\begin{array}{l}\text { Group-B (Induced) } \\
\text { number }\end{array}$} & $\%$ \\
\hline Vaginal delivery & $\mathbf{4 5}$ & $\mathbf{9 0 . 0}$ & $\mathbf{4 1}$ & $\mathbf{8 2 . 0}$ \\
Spontaneous delivery & 38 & 56.0 & 28 & 56.0 \\
Ventouse delivery & 05 & 10.0 & 8 & 16.0 \\
Forceps delivery & 02 & 4.0 & 5 & 10.0 \\
LSCS & $\mathbf{5}$ & $\mathbf{1 0 . 0}$ & $\mathbf{9}$ & $\mathbf{1 8 . 0}$ \\
\hline Total & $\mathbf{5 0}$ & $\mathbf{1 0 0 . 0}$ & $\mathbf{5 0}$ & $\mathbf{1 0 0 . 0}$ \\
\hline
\end{tabular}

Table 3 PROM- delivery interval (PDI)

\begin{tabular}{lllll}
\hline & Group- A (expectant) & & Group-B(Induced) & \\
Time & number & $\%$ & number & $\%$ \\
\hline$<12$ hours & 5 & 10.0 & 36 & 72.0 \\
12-24 hours & 35 & 70.0 & 11 & 22.0 \\
$>24$ hours & 10 & 20.0 & 3 & 6.0 \\
Total & 50 & 100.0 & 50 & 100.0 \\
\hline
\end{tabular}

Table 4 Maternal outcome

\begin{tabular}{|c|c|c|c|c|}
\hline & \multicolumn{2}{|c|}{ Group- A (expectant) } & \multicolumn{2}{|c|}{ Group-B(Induced) } \\
\hline & number & $\%$ & number & $\%$ \\
\hline Fever & 3 & 6.0 & $\mathrm{I}$ & 2.0 \\
\hline PPH & 5 & 10.0 & 6 & 12.0 \\
\hline Sepsis & 5 & 10.0 & 3 & 6.0 \\
\hline $\mathrm{Nil}$ & 37 & 74.0 & 40 & 80.0 \\
\hline Total & 50 & 100.0 & 50 & 100.0 \\
\hline
\end{tabular}


Table 5 Neonatal outcome

\begin{tabular}{|c|c|c|c|c|}
\hline & Group- A & & Group-B & \\
\hline & number & $\%$ & number & $\%$ \\
\hline Birth asphyxia & 14 & 28.0 & 8 & 16.0 \\
\hline Mild APGAR $<7$ & 10 & 20.0 & 6 & 12.0 \\
\hline Severe APGAR $<5$ & 4 & 8.0 & 2 & 4.0 \\
\hline Sepsis & 2 & 4.0 & 0 & 0.0 \\
\hline Stillbirth /early neonatal death & 0 & 0.0 & 0 & 0.0 \\
\hline Nil & 36 & 72.0 & 42 & 84.0 \\
\hline Total & 50 & 100.0 & 50 & 100.0 \\
\hline
\end{tabular}

\section{Discussion}

In our study, the women in both the Group A (expectant management group) and Group-B (Induced group) were comparable with respect to mean maternal age, gestational age, parity, educational status, socio-economic background, urban-rural distribution. Since their socio-demographic profile was similar, therefore, any difference in outcome in these two groups was primarily due to different management protocols and not due to demographic differences. In our study we observed that vaginal delivery occurred in $90 \%$ of Group A and $82.0 \%$ in Group-B (Induced) patients. A study by Shanti K et al. ${ }^{9}$ stated LSCS rate as $5.7 \%$ in the expected group as compared to $12 \%$ in active group. ${ }^{9}$ In another study by Suneela $\mathrm{K}^{1}$ there were $88.3 \%$ vaginal delivery in expectant group and $85.0 \%$ in active management group i.e. $11.7 \%$ LSCS rate in expectant group and $15 \%$ in induced group. ${ }^{1}$

In our study, we observed intrapartum pyrexia in 65 of expectant group (Group-A) versus $2 \%$ in Group -B (Induced group). Suneela $\mathrm{K}^{1}$ reported $3.3 \%$ pyrexia in induced group and $5 \%$ in expectant group. ${ }^{1}$ Sumaira $\mathrm{Y}^{10}$ (Peshawar) reported $2.4 \%$ in induced group \& $16 \%$ fever in expectant group. ${ }^{10}$ In our study, PPH occurred in $10 \%$ Group-A \& $12 \%$ Group-B, whereas Suneela $\mathrm{K}^{1}$ reported $6.7 \% \mathrm{PPH}$ in expectant group \& $10 \%$ in induced group. ${ }^{1}$ This result may be because of the fact that- induction of labour has a higher incidence of PPH. ${ }^{1,2}$

In our study, $28.0 \%$ babies in Group-A suffered from birth asphyxia (severe- $8 \%$ and mild- $20 \%$ ) as compared to $16.0 \%$ in Group- B (mild$12 \%$ \& severe $4 \%$ ). Neonatal sepsis was observed in $4 \%$ of Group A and in none ( $0 \%)$ in Group B. This may be attributed to the fact that there was a prolonged - delivery interval in Group A (expectant group). There were no stillbirths or early neonatal deaths in either groups. In her study, Suneela K et al. ${ }^{1}$ reported severe birth asphyxia requiring ventilation in $6.6 \%$ in the expectant group and neonatal sepsis was reported as $11.7 \%$ in the expectant group. ${ }^{1}$

Active management in cases of PROM at term has shown many benefits in terms of reducing the latent period and better maternal satisfaction. ${ }^{8}$ Induction of labour in such cases not only reduces the PROM-delivery interval but also reduces neonatal and maternal sepsis without much significant rise in LSCS rate. It is clear that nearly $70 \%$ of term PROM patients go into spontaneous labour without induction within 24 hours. The method of induction whether PGE1 (misoprostol) or oxytocin depends on Bishop's pre-induction score or cervical findings.

\section{Conclusion}

In our study we concluded that with induction of labour in patients with PROM at term, the PROM delivery interval was reduced along with significantly better maternal satisfaction and feto maternal outcome. The expectant group who underwent conservative management had higher maternal and fetal morbidity, sepsis, longer hospital and NICU stay causing anxiety and distress to both patients and clinicians. Therefore, in all patients presenting with premature rupture of membranes at term should be actively managed with induction of labour after assessing the cervical condition according to the Bishop's pre-Induction score so as to reduce the incidence of maternal and fetal sepsis and morbidity.

\section{Acknowledgments}

None.

\section{Conflicts of interest}

None.

\section{References}

1. Suneela K. Prelabour rupture of membranes at term: Expectant Management vs Induction of labour. Sch. $J$ App Med Sci. 2016;4(4D):1424-1427.

2. Gary Cunningham F et al. Normal Labour. Chapter 22, Mc Gram Hill publishers, William Obst- (24th edn), NY , USA. 2014;886-933,948.

3. Larrañaga-Azcárate C, Campo-Molina G, Pérez-Rodríguez AF,et al. Dinoprostone Vaginal slow release system compared to expectant management in the active treatment of PROM at term, impact on maternal and fetal outcome. Acta Obstet Gynecol Scand 2008. 2008;87(2):195-200.

4. Shah K, Doshi H. PROM at term: early induction versus expectant management. J Obstet Gynaecol India. 2012;62(2):172-175.

5. Duff P. PROM in term patients: Induction of labour versus expectant management. Clin Obstet Gynecol. 1998;41:883-891.

6. Kappa KA, Cetrulo CL, Knuppel RA, et al. PROM: conservative approach. Am J Obs Gyn. 1979;134(6):655-661.

7. Hoffmann RA Anthony J, Fawcus S. Oral misoprostol versus placebo in the management of PROM at term. Int $J$ of Gynec Obstet. $2001 ; 72(3): 215-221$

8. Term Prelabour rupture of membranes, The Royal Australian and New Zealand College of Obstetricians and Gynaecologists (C-Obs36):1-9.

9. Shanti K, G Prameela Devi, T Bharathi, et al. Comparative study of active vs expectant management and maternal and neonatal outcome in PROM. IOSR JDMSS. 2015;14(4):34-39.

10. Sumaira Yasmeen, Aiman Yasmin, Noor Nasir Khattak, et al. Active versus conservative management of PROM at term. $J$ Postgrad Med Inst. 2013;27(1):63-68. 